\title{
Health-Related Quality of Life in Patients with Multiple Endocrine Neoplasia Type 1
}

\author{
Rachel S. van Leeuwaarde ${ }^{a}$ Carolina R.C. Pieterman ${ }^{b}$ Anne M. May ${ }^{c}$ \\ Olaf M. Dekkers ${ }^{d}$ Anouk N. van der Horst-Schrivers ${ }^{e}$ Ad R. Hermus ${ }^{f}$ \\ Wouter W. de Herderg Madeleine L. Drent ${ }^{\text {h }}$ Peter H. Bisschop ${ }^{i}$ Bas Havekes ${ }^{j}$ \\ Menno R. Vriensk ${ }^{k}$ Gerlof D. Valk ${ }^{a}$ \\ ${ }^{a}$ Department of Endocrine Oncology, University Medical Center Utrecht, University of Utrecht, Utrecht, \\ The Netherlands; ${ }^{b}$ Dutch MEN advocacy group, Department of Endocrine Oncology, University Medical Center \\ Utrecht, University of Utrecht, Utrecht, The Netherlands; ' $D$ epartment of Clinical Epidemiology, Julius Center \\ for Health Sciences and Primary Care, University Medical Center Utrecht, University of Utrecht, Utrecht, \\ The Netherlands; ${ }^{\mathrm{d} D e p a r t m e n t s}$ of Endocrinology and Metabolism and Clinical Epidemiology, Leiden University

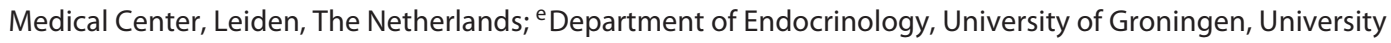 \\ Medical Center Groningen, Groningen, The Netherlands; ${ }^{f}$ Department of Endocrinology, Radboud University \\ Medical Center, Nijmegen, The Netherlands; ${ }^{9}$ Department of Internal Medicine, Erasmus Medical Center, \\ Rotterdam, The Netherlands; ${ }^{\text {h}}$ Department of Internal Medicine, Section of Endocrinology, VU University Medical \\ Center, Amsterdam, The Netherlands; 'Department of Endocrinology and Metabolism, Academic Medical Center, \\ Amsterdam, The Netherlands; ${ }^{j}$ Department of Internal Medicine, Division of Endocrinology, Maastricht University \\ Medical Center, Maastricht, The Netherlands; ${ }^{k}$ Department of Endocrine Surgery, University Medical Center Utrecht, \\ University of Utrecht, Utrecht, The Netherlands
}

\section{Keywords}

Multiple endocrine neoplasia type $1 \cdot$ Quality of life · Short Form 36 questionnaire

\begin{abstract}
Introduction: Multiple endocrine neoplasia type 1 (MEN1) is a hereditary endocrine tumor syndrome characterized by the triad of primary hyperparathyroidism, duodenopancreatic neuroendocrine tumors (pNETs), and pituitary tumors. Patients are confronted with substantial morbidity and are consequently at risk for an impaired quality of life (QOL). Meticulous assessment of QOL and associated factors in a representative population is needed to understand the full spectrum of the burden of the disease. Patients and Meth-
\end{abstract}

karger@karger.com www.karger.com/nen

Karger $\frac{1}{\%}$

GOPEN ACCESS
(C) 2020 The Author(s)

Published by S. Karger AG, Basel

This article is licensed under the Creative Commons AttributionNonCommercial-NoDerivatives 4.0 International License (CC BYNC-ND) (http://www.karger.com/Services/OpenAccessLicense). Usage and distribution for commercial purposes as well as any distribution of modified material requires written permission. ods: A cross-sectional study was performed using the national Dutch MEN1 cohort. Patients with a confirmed MEN1 mutation received the SF-36 Health Related Quality of Life questionnaire and questions regarding sociodemographic and medical history. Results: A total of 227 of 285 (80\%) eligible MEN1 patients returned the questionnaires. Health-related QOL scores (HRQOL) in MEN1 patients were significantly lower for the majority of subscales of the SF-36 in comparison with the general Dutch population. The most consistent predictor for HRQOL was employment status, followed by the presence of a pituitary tumor. $16 \%$ of patients harboring a pNET and $29 \%$ of patients with a pituitary tumor according to the medical records, reported that they were unaware of such a tumor. These subgroups of patients had several significant better QOL scores than patients who were 
aware of their pNET or pituitary tumors. Conclusion: Patients with MEN1 have an impaired QOL in comparison with the general Dutch population warranting special attention within routine care. For daily practice, physicians should be aware of their patients' impaired QOL and of the impact of unemployment on QOL.

(c) 2020 The Author(s)

Published by S. Karger AG, Basel

\section{Introduction}

Multiple endocrine neoplasia type 1 (MEN1) is a hereditary disease characterized by the classic triad of primary hyperparathyroidism ( $\mathrm{pHPT}$ ), pancreatic neuroendocrine tumors (pNETs), and pituitary tumors $[1,2]$. Other encountered neoplasms are neuroendocrine tumors of thymic, bronchial, or gastric origin, adrenal tumors, and smooth muscle, skin, and subcutaneous tumors [3]. Recently, it was reported that females with MEN1 have an almost 3 times higher risk for breast cancer at a 15 years younger age, which underlines the complexity and severity of the disease $[4,5]$.

The penetrance of the disease is high, especially for pHPT, pNETs, and pituitary tumors, respectively 100, 80, and $70 \%$, with the first manifestations occurring in childhood, further contributing to the burden of the disease for MEN1 mutation carriers [6, 7]. Confronted by this knowledge, accompanied by significant morbidity and early mortality, nowadays mostly arising from pNETs, MEN1 patients are at risk for impaired quality of life (QOL) $[8,9]$.

Recently it was reported that MEN1 patients have a high fear of MEN1-related tumor recurrence [8]. Studies assessing the health-related QOL (HRQOL) of patients with MEN1 indicate an impaired HRQOL, with a higher rate of depression for patients with a high burden of disease and treatment [9]. It is also suggested that HRQOL in MEN1 patients is worse than HRQOL in the general population $[9,10]$. Unfortunately, due to the manner of recruiting the participants, the studied populations were often prone for selection bias. Since these studies recruited in hospital patients or via patients' support groups, supposedly patients in need of medical or peer support were included. Therefore, the reported HRQOL might not be generalizable to the total MEN1 patient population. In addition, in several reports, medical information was assessed by self-reporting and there was no access to the medical records to retrieve the actual medical disease status. Therefore, it was unclear whether the reported disease status of patients was accurate $[9,11]$. In this respect, it is also not known whether these patients were actual genetically proven MEN1 patients or so-called "phenocopies," who have been reported to be a different disease entity with a more indolent disease course [6].

The Dutch MEN1 Study Group (DMSG) has meticulously registered the Dutch MEN1 population in a national database. This database contains data from 1990 up to present, collected every quarter of every year. The design of long follow-up and high density of the data allows for an accurate representation of disease status of all patients. This high coverage also minimizes the occurrence of a selected study sample [12].

The primary aim of this study was to assess HRQOL in the Dutch MEN1 cohort in order to compare the HRQOL with the general Dutch population and to assess which variables were predicators for worse HRQOL. The secondary aim was to evaluate if the self-reported MEN1 manifestations were in line with the disease status as reported in the medical records and whether a discrepancy affected the HRQOL.

\section{Methods}

\section{Study Population}

Participants were recruited from the Dutch MEN1 cohort. This MEN1 cohort is established by the DMSG. Participants were retrieved from the DMSG database. This longitudinal database includes $>90 \%$ of all Dutch MEN1 patients, aged 16 years and older at the end of 2017, treated at one of the Dutch University Medical Centers (UMCs) between 1990 up to present [12]. Patients were eligible for the present study if they had a confirmed MEN1 mutation. Demographic and clinical data such as MEN1-related medical history were retrieved from this database.

\section{Disease Manifestations}

pHPT was defined as hypercalcemia combined with elevated or inappropriately non-suppressed parathyroid hormone levels in 2 consecutive measurements. The presence of a pNET was confirmed according to the outcome of pathology examination. If pathological diagnosis was not available, pNET presence was based on MRI, computed tomography, or endoscopic ultrasound, which had to be confirmed at least once by consecutive imaging studies. Pathological diagnosis was generally not available in patients that were not eligible for surgery, for example, in case of a small tumor size. The absence of a pNET also had to be confirmed on a minimum of 2 subsequent imaging studies during follow-up. The reference standard for the presence of pituitary tumors was (1) pathology or (2) consecutive radiological examination demonstrating a pituitary tumor. Details for the reference standard of pHPT, pNET, and pituitary tumors have been described previously [13-15].

\section{Study Design}

A cross sectional study was conducted in which eligible patients were invited to complete a questionnaire from April 2015 till December 2017. After 2 weeks, a reminder e-mail was sent to the participants. The questionnaire could be completed by hand or as a 
web-based questionnaire. All participants provided written informed consent. Data from this cohort has been published earlier [8]. The Medical Ethical Committees (MERC) of all UMCs in the Netherlands confirmed that the Medical Research Involving $\mathrm{Hu}-$ man Subjects Act (WMO) did not apply for this study and therefore, an official approval was not required under WMO.

\section{Questionnaires}

Sociodemographic data such as education and employment were obtained.

Disease Status. Participants had to complete questions on the presence of their own history of pHPT, pituitary tumors, and pNETs. In addition they were asked if they were operated or received other treatments for these manifestations. If they were operated the exact year of surgery was asked.

Health-Related Quality of Life. HRQOL was assessed using the Short Form 36 (SF-36) Health Survey composed of 8 multiitem scales assessing physical functioning, role limitations due to physical health problems and emotional problems, bodily pain, general health perceptions, vitality, social functioning, and general mental health. Scale scores range from 0 to 100 , with higher scores indicating better levels of functioning and wellbeing. Cronbach's a for the SF-36 scales ranged from 0.84 (social functioning) to 0.93 (physical pain). Only general health perception had a low Cronbach's a (0.55), which will therefore be of low significance.

The normative data on the SF-36 Health Survey were derived from the general population of the Netherlands [16].

\section{Statistical Analysis}

Descriptive statistics were applied to characterize the study population. Univariate analyses (independent sample $t$ test/MannWhitney U test, chi-square test/Fisher's exact test, Pearson correlation) were used when appropriate and to evaluate which MEN1related manifestations and sociodemographic variables were associated with HRQOL.

A multivariable analysis adjusted for age and gender was carried out using multiple linear regression to assess which patient characteristics were associated with HRQOL. Collinearity was tested using variance inflation factors (VIF). In the linear models, none of the VIF values were $>1.6$, suggesting that collinearity was not a problem.

Analyses were conducted using SPSS 22.0. $p$ values $<0.05$ were considered as statistically significant.

\section{Results}

\section{Response Rate}

Between 2015 and 2017, a total of 285 patients (120 men and 165 women) were eligible for inclusion, of which 252 (102 men and 150 women) received the questionnaire. Thirty-three patients could not be reached. The questionnaire was completed by 227 individuals ( 84 [70\%] men and 143 [87\%] women), resulting in a total response rate of $80 \%$ [8].
Table 1. Baseline characteristics $(n=227)$

$\begin{array}{lc}\text { Gender } & \\ \text { Female } & 143(63 \%) \\ \text { Male } & 84(37 \%) \\ \text { Age, years, mean (SD) } & 47(15) \\ \text { Education } & \\ \quad \text { Primary school } & 6(3 \%) \\ \quad \text { Secondary school } & 149(65 \%) \\ \quad \text { College or university } & 66(29 \%) \\ \text { Employment (age }<65 \text { years) } & \\ \quad \text { Yes } & 154(80 \%) \\ \quad \text { No } & 39(20 \%) \\ \text { Index case } & \\ \quad \text { Yes } & 51(23 \%) \\ \text { No } & 173(76 \%) \\ \text { Presymptomatic diagnosis } & \\ \quad \text { Yes } & 98(44 \%) \\ \text { No } & 125(56 \%) \\ \text { Years since MEN1 diagnosis } & \\ \quad<5 \text { years } & 27(12 \%) \\ \geq 5 \text { years } & 190(84 \%)\end{array}$

The data presented here have been published previously [8]

\section{Population Characteristics}

The mean age of the study population was $47(\mathrm{SD} \pm 15)$ years (Table 1). This cohort has been described and the data presented in Table 1 has been published previously [8].

All patients had a confirmed MEN1 mutation, or one or more MEN1-related manifestations and a first-degree relative with MEN1. Eighty-three percent of patients reported ever having had a pPHT. More than half of the patients reported ever having had a pNET (55\%) and 38\% of patients had a self-reported pituitary tumor.

\section{Health-Related Quality of Life}

HRQOL scores in MEN1 patients, adjusted for age and gender, were significantly lower for the majority of subscales of the Health Related Quality of Life SF-36 in comparison with the general Dutch population (Fig. 1). The subscales general health perceptions and vitality were 0.5 SD lower, which can be considered as a clinically relevant difference. The only scale that was comparable with the general population was the physical functioning scale.

\section{Self-Reported Manifestations versus Manifestations according to the Medical Records}

Only one (3\%) of 31 patients who, according to the medical records, did not have a pHPT reported having a pHPT. A total of 7 (4\%) of 192 patients with pHPT 
Fig. 1. Mean SF-36 scores of MEN1 patients versus the general Dutch population. $* p<0.05$.
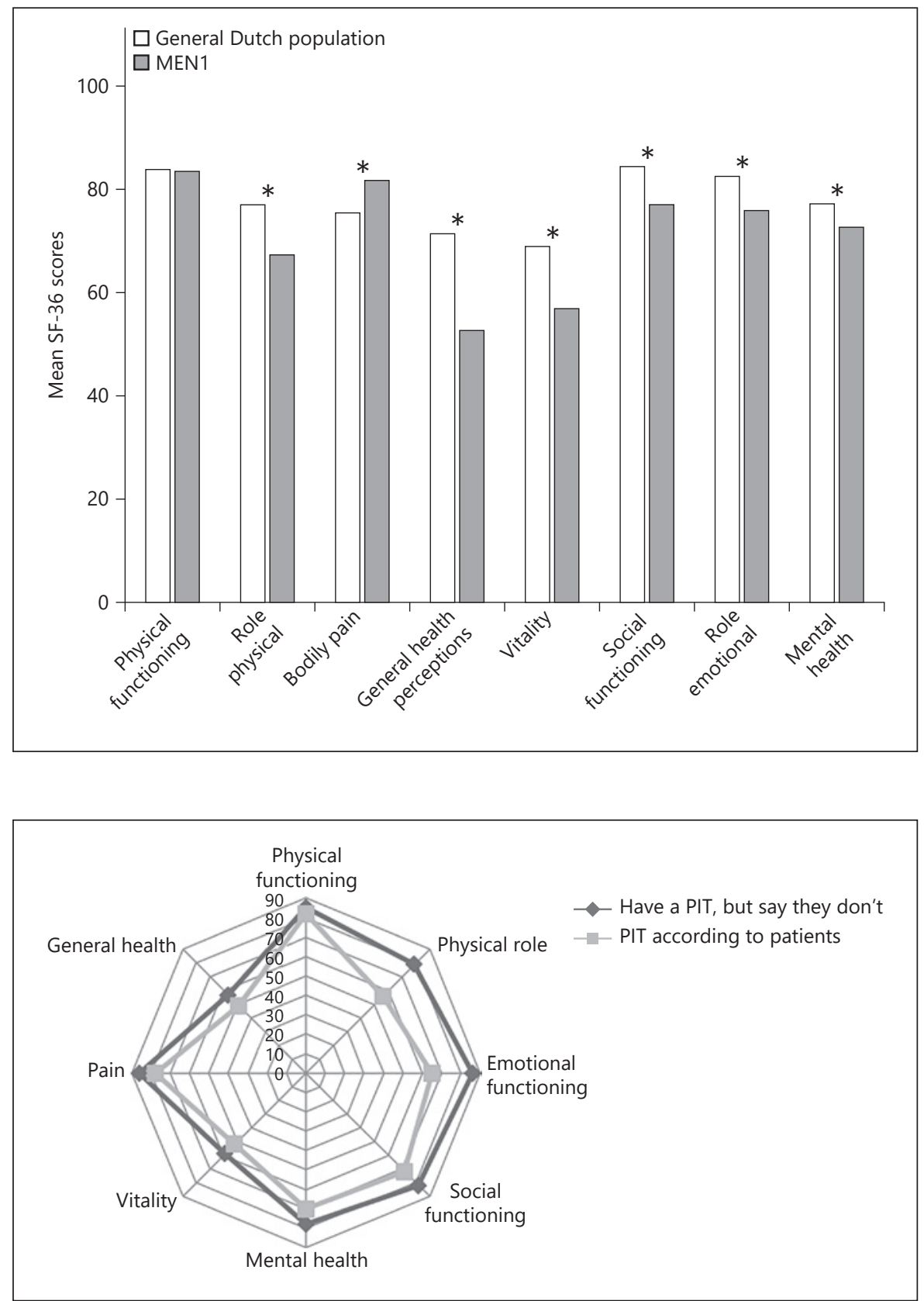

Fig. 2. HRQOL scores of patients with a pituitary tumor (PIT) in comparison with patients who do not think they have a PIT, but they do according to the medical data. Physical role $(p=0.01)$, emotional functioning $(p=0.02)$, and mental health $(p=$ 0.02).
Six (5\%) of the 122 patients who according to the medical records did not have and never had a pNET, reported having a pNET. Twenty-nine (29\%) of 101 patients who ever had a pituitary tumor according to the medical records, reported that they did not have and never had a pituitary tumor. The median size of the pituitary tumors in this group was $5 \mathrm{~mm}$ (IQR 4-6) compared to $5 \mathrm{~mm}$ (IQR 3-7) in patients who accurately reported having a pituitary tumor. One of those patients underwent a trans- 
Table 2. Characteristics of MEN1 patients of working age and independent variables of employment

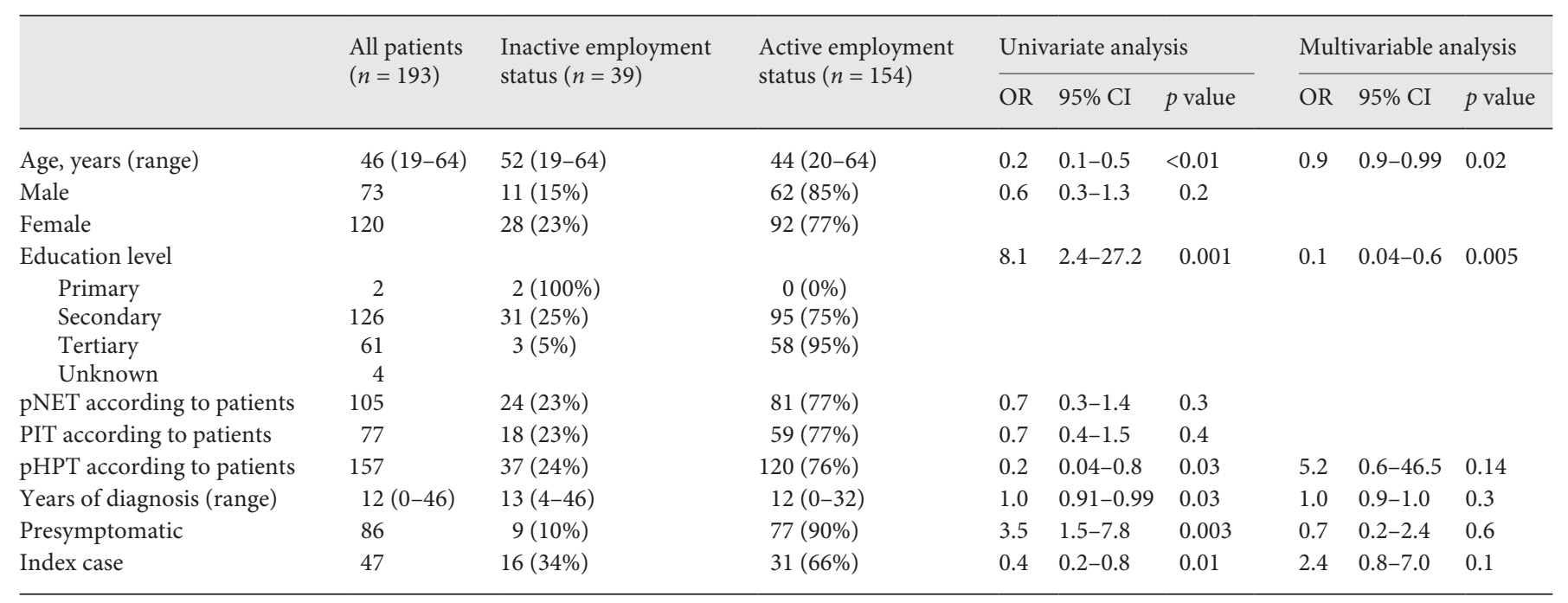

pNET, pancreatic neuroendocrine tumor; PIT, pituitary tumor; pHPT, primary hyperparathyroidism.

sphenoidal resection for a pituitary tumor. Four (5\%) of 85 patients who never had a pituitary tumor thought they did have a pituitary tumor.

Patients who reported not having a pNET, but had a pNET according to the medical records, had slightly better HRQOL scores in comparison with patients who reported ever having had a pNET. This difference was significant for the emotional functioning score, with mean scores of 85.9 and 65.4 , respectively (CI -35.4 to $-5.6 ; p=0.03$ ).

Patients who ever had a pituitary tumor diagnosis as reported in their medical records, but reported not having a pituitary tumor, had better HRQOL scores than patients who reported having or having had a pituitary tumor (Fig. 2). This difference was significant for the physical role $(p=0.01)$, emotional functioning $(p=0.02)$, and mental health $(p=0.02)$ subscale.

\section{Univariate Analysis}

In univariate analyses, age, years since MEN1 diagnosis, education level, pituitary tumor, index cases, and employment were significantly related to 2 or more subscales of the Health Related Quality of Life SF-36 (online suppl. data; see www.karger.com/doi/10.1159/000508374).

\section{Multivariable Analysis}

The multivariable analyses included age, the presence of a pNET, pHPT, pituitary tumor, years of diagnosis, education level, index case, employment (yes/no), and presymptomatic diagnosis (yes/no). The employment status, being an index case, and a pituitary tumor diagnosis were associated with HRQOL. Employment was the only predictor that was significantly related to all subscales, except for the emotional functioning scale (online suppl. data).

\section{Employment Status}

A subgroup analysis of patients before the retirement age was conducted since employment appeared to be such a crucial factor for HRQOL. Therefore, the group was divided according to employment status: employed $(n=154)$ and unemployed $(n=39$; Table 2$)$.

Age at completion of the questionnaire and education level were significantly different between the employed and unemployed group. The mean age of patients in the employed group was almost 10 years younger than the unemployed group; 42 and 51 years, respectively $(p=$ $0.01)$. Patients with a college or university degree $(n=61)$ had the highest percentage of employment $(95 \%, n=58)$ in comparison with patients with only primary education ( $n=2$, who were unemployed) or secondary education ( $n=126$, of whom 95 are employed; $75 \% ; p=0.01$ ). Odds ratios and $95 \% \mathrm{CI}$ of univariate and multivariable analyses of active versus inactive employment status are presented in Table 2.

The multivariable analysis of patients below 65 years of age with the same variables as reported earlier showed the same outcomes, concluding that employment remained a crucial predictor for HRQOL in patients with MEN1 (Table 2). 


\section{Discussion}

In this large nationwide study including a representative sample of MEN1 patients, QOL was significantly and clinically relevantly impaired in comparison with the general Dutch population. A worse HRQOL in MEN1 patients was suggested in previous studies with either selected or small MEN1 populations [9, 10].

The present population revealed no difference in physical functioning in comparison with the general population. However, reduced physical functioning compared with chronic conditions and the general population was found in a cohort part of the American MEN support group [17]. The discrepancy might be explained by a selection of patients that experience (physical) impairment caused by the disease and therefore might have sought support in this group. Recruiting patients via support groups might result in a selection of patients and therefore result in different findings in comparison with a population-based study.

On the contrary, for other endocrine cancer syndromes such as VHL and MEN2, HRQOL was comparable with the general population. This is striking, because both syndromes have considerable morbidity [18, 19].

An in-depth analysis revealed that the most consistent predictor for HRQOL was employment status, followed by the presence of a pituitary tumor. Employment was a robust predictor for HRQOL across all HRQOL subscales. Employment has proven to be beneficial for health in general [20]; therefore, not being able to work can have a significant effect on the HRQOL. The percentage of unemployment was $20 \%$ in this population. Unemployment in the Netherlands was approximately 5.5\% during the time of this study, revealing a high percentage of unemployment in MEN1 patients.

An online survey in $153 \mathrm{MEN} 1$ patients who are part of the American MEN support group revealed that this group experienced significant financial burden and unemployment, which were both correlated to worse HRQOL [17]. A similar effect of employment on HRQOL is observed in different malignancies [21]. In addition, in a MEN2 cohort, a higher physical functioning score was observed in actively working patients than in inactive subjects [19].

Both, losing a job because of health issues and being a long-term cancer survivor are risk factors for lower HRQOL [22]. Cancer survivors who continue to work have a better health and HRQOL than patients who are not able to work [23]. Since cancer survivorship is associ- ated with unemployment, this group of patients is at risk of not returning to work [24]. In our study, an older age and lower levels of education were associated with unemployment. This is in line with previous studies assessing predictors of unemployment of cancer survivors [25]. QOL and return to work seem to benefit from rehabilitation programs [26]. Multidisciplinary interventions that combine vocational counselling, patient education/counselling, and physical exercises showed higher return to work rates than care as usual [24]. Intervention studies assessing a similar rehabilitation program in MEN1 patients would be helpful to gain more insight in the value of these programs and to develop a MEN1-specific multidisciplinary reactivation program.

Remarkably, in a substantial proportion of patients, there was a discrepancy between the self-reporting of patients of having a pNET or pituitary tumor and the disease status as reported in their medical records. In 16 and $29 \%$ of patients who reported not having a pNET or pituitary tumor, the medical records showed the presence of these manifestations. Patients could not have been informed properly or could have forgotten their own disease status or misunderstood the questions. Interestingly, patients who were not aware of having a pNET or a pituitary tumor had a better HRQOL compared to patients who were aware of their disease status. When compared with patients having a pNET, the differences of HRQOL between those who were aware compared to those unaware, was worse in patients having a pituitary tumor. This suggests that in particular the knowledge of patients that they have a pituitary tumor played a significant role in the HRQOL.

This finding also suggests that cognitive avoidance can be present. This is a coping mechanism that functions as a psychological defense and has been described in a MEN2 population, primarily occurring in cured patients [19]. Most MEN1-related tumors remain stable and asymptomatic, and require no treatment. In these cases, the major burden is the knowledge about the disease status without a direct clinical consequence. This requires specific coping strategies in dealing with lifelong disease burden with an insecure outcome necessitating specific attention.

\section{Limitations}

The cross-sectional design of the study does not allow assessing the HRQOL in the course of the disease. A longitudinal study would give insight into the variability of HRQOL in the course of MEN1-related therapies and surveillance. The heterogeneity of MEN1, with its variable disease prevalence, various treatment options, and 
strict follow-up regimen sets the ideal basis for a longitudinal HRQOL study. Long-term distress is more likely to occur in this population than short-term distress. This was also seen in a MEN2 cohort with relatively more depression and anxiety [19].

\section{Strengths}

A major strength is the high response rate of $80 \%$ of this study contributing to the validity and generalizability of the study. Another major strength is the availability of the DMSG longitudinal MEN1 database comprising an extensive clinical dataset. Data filled out by the respondents could therefore be cross-referenced from this database.

\section{Clinical Implications}

Because of the reduced HRQOL, special attention of care providers for this aspect should be routine in the regular care for patients with MEN1. Integrating structured HRQOL assessments to the surveillance program will provide more insight into the perceived burden of patients with MEN1 and the HRQOL in the course of the disease. This will ultimately contribute to improving the quality of this aspect of MEN1 patient care. Management of MEN1 patients in a multidisciplinary team, preferably including a psychologist, can be of benefit for this population.

Patient counselling on the natural course of the disease and possible treatment options are imperative to provide insight into the disease. Tumors have malignant potential, but genetic testing to pursue early diagnosis and subsequent surveillance has decreased morbidity and mortality [27]. This information might assure patients to adhere to the current screening protocols and be assured that an early diagnosis will lead to an improved overall outcome. To prevent the discordance between patients' self-reported and actual disease status, physicians should pay special attention to the patients' understanding of the disease and review whether their disease insight is accurate.

Physicians should be especially aware of the impact of the relationship between unemployment and HRQOL. Unemployed patients should be considered as high-risk patients for worse HRQOL. Multidimensional rehabilitation programs might be helpful in returning to work and hereby improving HRQOL.

\section{Conclusions}

In this, up to now largest unselected MEN1 cohort, patients with MEN1 have an impaired QOL in comparison with the general Dutch population. Unemployment and having a pituitary tumor are consistent predictors for a worse QOL. Awareness of patients' actual disease status influences their QOL. Lack of awareness results in better QOL. For daily practice, physicians should be aware of their patients' impaired QOL and the impact of unemployment on the QOL. A multidisciplinary approach in this population is warranted and will benefit the care for patients with MEN1.

\section{Statement of Ethics}

The Medical Ethical Committees (MERC) of all UMCs in the Netherlands confirmed that the Medical Research Involving Human Subjects Act (WMO) did not apply for this study and therefore, an official approval was not required under WMO.

\section{Conflict of Interest Statement}

The authors have no conflicts of interest to declare.

\section{Funding Sources}

There are no funding sources to declare.

\section{Author Contributions}

R.S.v.L. designed the study, did the data acquisition and analysis, interpreted the data, and drafted the work. C.R.C.P. and A.M.M. co-designed the study. O.M.D., A.R.H., A.N.v.d.H.-S., W.W.d.H., B.H., M.L.D., and P.H.B. collected the data and substantially revised the work. M.R.V. co-analyzed the data. G.D.V. co-designed the study, interpreted the data, and drafted the work.

\section{References}

1 Chandrasekharappa SC, Guru SC, Manickam $\mathrm{P}$, Olufemi SE, Collins FS, Emmert-Buck MR, et al. Positional cloning of the gene for multiple endocrine neoplasia-type 1 . Science. 1997 Apr;276(5311):404-7.

2 Kouvaraki MA, Lee JE, Shapiro SE, Gagel RF, Sherman SI, Sellin RV, et al. Genotype-phenotype analysis in multiple endocrine neoplasia type 1. Arch Surg. 2002 Jun;137(6):641-7.

3 Goudet P, Murat A, Binquet C, CardotBauters C, Costa A, Ruszniewski P, et al. Risk factors and causes of death in MEN1 disease. A GTE (Groupe d'Etude des Tumeurs Endocrines) cohort study among 758 patients. World J Surg. 2010 Feb;34(2):249-55.

4 Dreijerink KM, Goudet P, Burgess JR, Valk GD; International Breast Cancer in MEN1 Study Group. Breast-cancer predisposition in multiple endocrine neoplasia type $1 . \mathrm{N}$ Engl Med. 2014 Aug;371(6):583-4. 
5 Ito $\mathrm{T}$, Igarashi $\mathrm{H}$, Uehara $\mathrm{H}$, Berna $\mathrm{MJ}$, Jensen RT. Causes of death and prognostic factors in multiple endocrine neoplasia type 1: a prospective study: comparison of 106 MEN1/ Zollinger-Ellison syndrome patients with 1613 literature MEN1 patients with or without pancreatic endocrine tumors. Medicine (Baltimore). 2013 May;92(3):135-81.

6 de Laat JM, van der Luijt RB, Pieterman CR, Oostveen MP, Hermus AR, Dekkers OM, et al. MEN1 redefined, a clinical comparison of mutation-positive and mutation-negative patients. BMC Med. 2016 Nov;14(1):182.

7 Goudet P, Dalac A, Le Bras M, Cardot-Bauters C, Niccoli P, Lévy-Bohbot N, et al. MEN1 disease occurring before 21 years old: a 160-patient cohort study from the Groupe d'étude des Tumeurs Endocrines. J Clin Endocrinol Metab. 2015 Apr;100(4):1568-77.

8 van Leeuwaarde RS, Pieterman CR, Bleiker EM, Dekkers OM, van der Horst-Schrivers AN, Hermus AR, et al. High fear of disease occurrence is associated with low quality of life in patients with Multiple Endocrine Neoplasia type 1: Results from the Dutch MEN1 Study Group. J Clin Endocrinol Metab. 2018 Jun;103(6):2354-61.

9 Peipert BJ, Goswami S, Yount SE, Sturgeon C. Health-related quality of life in MEN1 patients compared with other chronic conditions and the United States general population. Surgery. 2018 Jan;163(1):205-11.

10 Berglund G, Lidén A, Hansson MG, Öberg K, Sjöden PO, Nordin K. Quality of life in patients with multiple endocrine neoplasia type 1 (MEN 1). Fam Cancer. 2003;2(1):27-33.

11 Goswami S, Peipert BJ, Helenowski I, Yount SE, Sturgeon C. Disease and treatment factors associated with lower quality of life scores in adults with multiple endocrine neoplasia type. Surgery. 2017 Dec;162(6):1270-7.

12 de Laat JM, Tham E, Pieterman CR, Vriens MR, Dorresteijn JA, Bots ML, et al. Predicting the risk of multiple endocrine neoplasia type 1 for patients with commonly occurring endocrine tumors. Eur J Endocrinol. 2012 Aug; 167(2):181-7.
13 de Laat JM, Dekkers OM, Pieterman CR, Kluijfhout WP, Hermus AR, Pereira AM, et al. Long-Term Natural Course of Pituitary Tumors in Patients With MEN1: Results From the DutchMEN1 Study Group (DMSG). J Clin Endocrinol Metab. 2015 Sep;100(9): 3288-96.

14 Pieterman CR, van Hulsteijn LT, den HM, van der Luijt RB, Bonenkamp JJ, Hermus AR, et al. Primary hyperparathyroidism in MEN1 patients: a cohort study with longterm followup on preferred surgical procedure and the relation with genotype. Ann Surg. 2012 Jun; 255(6):1171-8

15 Pieterman CR, de Laat JM, Twisk JW, van Leeuwaarde RS, de Herder WW, Dreijerink $\mathrm{KM}$, et al. Long-term natural course of small nonfunctional pancreatic neuroendocrine tumors in MEN1-results from the Dutch MEN1 study group. J Clin Endocrinol Metab. 2017 Oct;102(10):3795-805.

16 Aaronson NK, Muller M, Cohen PD, EssinkBot ML, Fekkes M, Sanderman R, et al. Translation, validation, and norming of the Dutch language version of the SF-36 Health Survey in community and chronic disease populations. J Clin Epidemiol. 1998 Nov;51(11):1055-68.

17 Peipert BJ, Goswami S, Helenowski I, Yount $\mathrm{SE}$, Sturgeon C. Financial burden is associated with worse health-related quality of life in adults with multiple endocrine neoplasia type 1. Surgery. 2017 Dec;162(6):1278-85.

18 Lammens CR, Bleiker EM, Verhoef S, Hes FJ, Ausems MG, Majoor-Krakauer D, et al. Psychosocial impact of Von Hippel-Lindau disease: levels and sources of distress. Clin Genet. 2010 May;77(5):483-91.

19 Rodrigues KC, Toledo RA, Coutinho FL, Nunes AB, Maciel RM, Hoff AO, et al. Assessment of Depression, Anxiety, Quality of Life, and Coping in Long-Standing Multiple Endocrine Neoplasia Type 2 Patients. Thyroid. 2017 May;27(5):693-706.
20 van der Noordt M, IJzelenberg H, Droomers M, Proper KI. Health effects of employment: a systematic review of prospective studies. Occup Environ Med. 2014 Oct;71(10):730-6.

21 Magyari F, Kósa K, Berecz R, Illés A, Miltényi $Z$, Simon Z, et al. Employment status and health related quality of life among Hodgkinlymphoma survivors' - results based on data from a major treatment center in Hungary. Health Qual Life Outcomes. 2017 Sep;15(1): 180.

22 Lee SY, Kim SJ, Shin J, Han KT, Park EC. The impact of job status on quality of life: general population versus long-term cancer survivors. Psychooncology. 2015 Nov;24(11): $1552-9$.

23 Duijts SF, Kieffer JM, van Muijen P, van der Beek AJ. Sustained employability and healthrelated quality of life in cancer survivors up to four years after diagnosis. Acta Oncol. 2017 Feb;56(2):174-82.

24 de Boer AG, Taskila T, Ojajärvi A, van Dijk FJ, Verbeek JH. Cancer survivors and unemployment: a meta-analysis and meta-regression. JAMA. 2009 Feb;301(7):753-62.

25 Mehnert A, de Boer A, Feuerstein M. Employment challenges for cancer survivors. Cancer. 2013 Jun;119 Suppl 11:2151-9.

26 Leensen MC, Groeneveld IF, Heide IV, Rejda T, van Veldhoven PL, Berkel SV, et al. Return to work of cancer patients after a multidisciplinary intervention including occupational counselling and physical exercise in cancer patients: a prospective study in the Netherlands. BMJ Open. 2017 Jun; 7(6):e014746.

27 van Leeuwaarde RS, van Nesselrooij BPM, Hermus AR, Dekkers OM, de Herder WW, van der Horst-Schrivers AN, et al. Impact of delay in diagnosis in outcomes in MEN1: results from the Dutch MEN1 study group. J Clin Endocrinol Metab. 2016 Mar;101(3): 1159-65. 